\title{
Expression of PRR11 protein and its correlation with pancreatic cancer and effect on survival
}

\author{
SONG TAN, ZHONGHUA JIANG, AIHUA HOU, JIHUA WANG, JINBO ZHANG and LINGLING DAI \\ Department of Oncology, Yantai Hospital of Traditional Chinese Medicine, Yantai, Shandong 264001, P.R. China
}

Received October 19, 2016; Accepted February 9, 2017

DOI: $10.3892 / 01.2017 .5974$

\begin{abstract}
This study aimed at finding the relationship between the level of expression of the PRR11 protein in pancreatic carcinoma, and the clinical characteristics of the tumor. PCR technique was used to analyze the expression levels of the PRR11 gene in 38 samples from pancreatic cancer patients and 10 samples from normal pancreatic tissues. Western blot analysis and immunohistochemistry were used to measure the expression of the PRR11. Additionally, the migration ability of cancerous cells expressing PRR11 and those with inhibited expression were compared using a wound healing assay. Finally, the relationships between the expression level of PRR11 protein and variables such as tumor size, tumor invasion, TNM stages, and the overall survival time of patients with pancreatic cancer were calculated. Our results showed the expression level of the PRR11 gene in pancreatic cancer tissues was significantly higher than that in normal pancreatic tissues. The detection of PRR11 protein in cancer tissues versus normal tissues was $78.9(30 / 38)$ vs. $0(0 / 10)$, respectively. The western blot results confirmed this by showing a significantly higher level of expression of the PRR11 protein in pancreatic cancer tissues than in normal tissues $(\mathrm{P}<0.05)$. Inhibiting the expression of PRR11 in cancer cells reduced the migration ability of the cells. Finally, the expression of PRR11 was positively correlated with the invasion, disease and tissue differentiation stages of the pancreatic cancer. By comparing clinical data and expression patterns in patients, we found the survival rate in those expressing the PRR11 protein by immunohistochemistry to be lower than in those with tissues negative to the PRR11 protein. Our results show, the expression of PRR11 protein in pancreatic cancer is closely related to the development of the cancer and a poor prognosis. These findings provide a theoretical and experimental basis for
\end{abstract}

Correspondence to: Dr Lingling Dai, Department of Oncology, Yantai Hospital of Traditional Chinese Medicine, 39 Xingfu Road, Yantai, Shandong 264001, P.R. China

E-mail:db0g95@163.com

Key words: PRR11 protein, pancreatic cancer, prognosis approaching the diagnosis and treatment of pancreatic cancer using PRR11 as a molecular target.

\section{Introduction}

Among malignant tumors, pancreatic cancer occurs frequently and is one of the most severe gastrointestinal tumors with extremely high mortality rates (1). Due to the anatomical location of the pancreas, and the fact that tumors are found in advanced stages, the chance of performing a curative surgery is small. Relevant data have shown a relatively serious recurrence rate in patients within 5 years after operation, and a survival rate of $<5$ years for up to $\sim 95 \%$ of them (2). Pancreatic cancer markedly reduces the living quality of patients, but also causes a heavy economic burden on society. The complexity of pancreatic cancer and its poorly understood pathogenesis, are factors determining the difficulty in achieving a prompt diagnosis and an effective treatment. With the development of more advanced molecular biology techniques, the search for therapeutic targets is currently very active with the aim of finding a specific and effective treatment alternative.

PRR11 is a newly found gene, located at chromosome $17 q 22$ (3). Studies have shown this gene is an evolutionarily conserved one, which may play a role in cell proliferation (4). Furthermore, PRR11 is upregulated in various tumors, including brain, lung, breast and ovarian cancer tumors; and its high expression has been related to the development, deterioration and poor prognosis of various tumors (5-7). Nevertheless, the relationship between PRR11 and pancreatic cancer remains unclear. This study aimed to investigate the effects of PRR11 on pancreatic cancer.

\section{Materials and methods}

Patients, materials and methods. In this study, paraffin-embedded tissue samples were collected from a total of 48 patients who underwent pancreas operation in Yantai Hospital of Traditional Chinese Medicine from October 2012 to March 2014. The survival time of the patients was counted from the day of enrollment in the study to the time of death or the date of last collection of information in March, 2016. Network telephone surveys and out-patient re-examinations were used as means of evaluating the status of patients. This study was approved by the Ethics Committee of Yantai Hospital of Traditional Chinese Medicine. Signed written 
Table I.RT-PCR primer sequences and gene silencing sequences.

\begin{tabular}{ll}
\hline Genes & \multicolumn{1}{c}{ Sequences } \\
\hline PRR11 & F: 5'-TGCGTGTGGAGTATTTGGATG-3' \\
& R: 5'-TGGTACAGTCAGAGCCAACCTC-3' \\
\multirow{2}{*}{-actin } & F: 5'-CAGCCATGTACGTTGCTATCCAGG-3' \\
& R: 5'-AGGTCCAGACGCAGGATGGCATG-3'
\end{tabular}

F, forward; R, reverse.

informed consents were obtained from all participants and/or guardians before the study.

The essential reagents used for this study include a rabbit polyclonal PRR11 antibody (dilution, 1:500; Sigma-Aldrich China, Inc., Shanghai, China), rabbit streptavidin-HRP kit and hematoxylin (both from Huaxia Yuanyang Science and Technology, Beijing, China), neutral balsam (Zhengheng Chemical Glass Instrument, Linyi, China), PBS solution (Huaxia Yuanyang Science and Technology), and alcohol (Zhengheng Chemical Glass Instrument). Instruments included an optical microscope (Beijing PDV Instrument Co., Ltd., Beijing, China), a micropipettor (Taixing Aibo Glassware Co. Ltd., Jiangsu, China), a fluorescence microscope, a supercentrifuge, and an ABI7900 PCR amplifier (all from Beijing PDV Instrument Co., Ltd.).

Cell culture. The cell strain Capan-1 of human pancreatic cancer purchased from CoBioer Biosciences Co., Ltd. (Nanjing, China) was cultured in 10\% fetal calf serum with high glucose Dulbecco's modified Eagle's medium (DMEM) culture solution at constant temperature $\left(37^{\circ} \mathrm{C}\right)$ in a cell culture incubator. The culture medium was replaced every two days (or as needed). Serial sub-cultivation was performed once the cells entered into the exponential growth phase.

Extraction of RNA in pancreatic cancer cells and PRRII $m R N A$ levels. mRNA expression levels were measured in tissues of 40 cases of pancreatic cancer and 8 normal pancreas controls. Taking SYBR as fluorescent dye and $\beta$-actin (ACTB) as the internal reference mRNA, the quantity of PRR11 mRNA expression was detected. The PRR11 primer sequences and GAPDH primer sequences are shown in Table I.

Expression of PRRI1 protein detected by western blot analysis. Frozen tissue samples stored in liquid nitrogen were cut into pieces with scissors. Each sample was homogenized in lysis buffer at a ratio of 1:20 w/v. After a centrifugation at $10,000 \mathrm{xg}$ for $20 \mathrm{~min}$ step the supernatant was used to measure the total protein. The quantification of the PRR11 protein was made following standard western blot analysis and normalizing to the internal reference $\beta$-actin.

Expression of PRRI1 protein detected by immunohistochemistry. Stored prepared paraffin sections were dewaxed. After washing with a $3 \%$ hydrogen peroxide aqueous solution, citric acid buffer solution (both from Biosharp, Hefei, China) was added and the samples were heated for renaturation. After

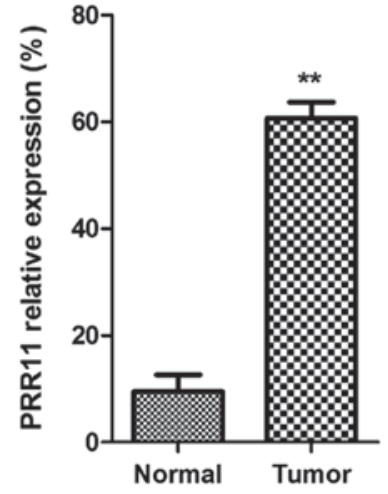

Figure 1. Pancreatic cancer detected by RT-PCR. Comparison between the expression of PRR11 in normal pancreatic tissues and that of pancreatic cancer group, ${ }^{* *} \mathrm{P}<0.01$.

blocking in goat serum, the primary antibody (dilution, 1:200; cat. no. SAB1102068; Sigma-Aldrich China, Inc.) was added and the samples incubated at $4^{\circ} \mathrm{C}$ overnight. Next day the samples were washed, and then the secondary goat anti-rabbit (HRP) IgG antibody (dilution, 1/2,000; cat. no. ab6721) was added and incubation at $37^{\circ} \mathrm{C}$ for $1 \mathrm{~h}$. Color development and re-dyeing were conducted after washing. Finally, observations were conducted under a microscope (Nikon, Tokyo, Japan).

Scoring of tissue samples was done by calculating the number of positive cells in each field of view. Scores were divided into four levels. The first level (scored as 0 ) contained $<5 \%$ of positive cells. The second level (scored as 1) referred to $<40 \%$ of positive cells. The third level (scored as 2 ) referred to $>40 \%$ of positive cells. The fourth and highest level (scored as 3) referred to $>60 \%$ positive cells. After statistical analysis, the above scoring results were divided into two groups. A low expression group of PRR11 for levels I and II, and a high expression group of PRR11 for levels III and IV. The final results were used in the statistical analyses.

Cell wound healing assay. The cell strains pPRR11-sh (inhibiting the expression of PRR11) and pControl-sh (expressing an empty control vector) were constructed and used in a wound healing assay. Firstly, the stable cell strains were inoculated on a 6-well plate, respectively. Cultivation was conducted under standard conditions for $12 \mathrm{~h}$. A separation glass needle was used to draw a thin line through the cell layer in each one of the plates. Dislodged cells were washed away, and fresh culture medium without serum was added, before placing the plates back in the incubator for a total of $48 \mathrm{~h}$.

Statistical analysis. Experimental data processing was tested using the SPSS 19.0 statistical software (IBM, Armonk, NY, USA). The difference in the expression level of PRR11 protein in the pancreatic cancer group and the normal group was calculated. Survival analysis was conducted for clinical prognosis data. $\mathrm{P}<0.05$ was considered to indicate a statistically significant difference.

\section{Results}

Changes of PRR11 mRNA in pancreatic cancer tissues. As shown in Fig. 1, mRNA expression of PRR11 in pancreatic 
Table II. Expression of PRR11 protein in pancreatic cancer and in normal pancreatic tissues.

\begin{tabular}{lcccccr}
\hline Tissues & Cases & $\begin{array}{c}\text { Positive } \\
\text { immunohistochemistry }\end{array}$ & $\begin{array}{c}\text { Negative } \\
\text { immunohistochemistry }\end{array}$ & $\begin{array}{c}\text { Positive } \\
\text { rate (\%) }\end{array}$ & $\chi^{2}$ & P-value \\
\hline $\begin{array}{l}\text { Pancreatic } \\
\text { cancer tissues }\end{array}$ & 38 & 30 & 8 & 78.9 & 32.047 & $\mathrm{P}<0.05$ \\
Normal tissues & 10 & 0 & 10 & 0 & & \\
\hline
\end{tabular}

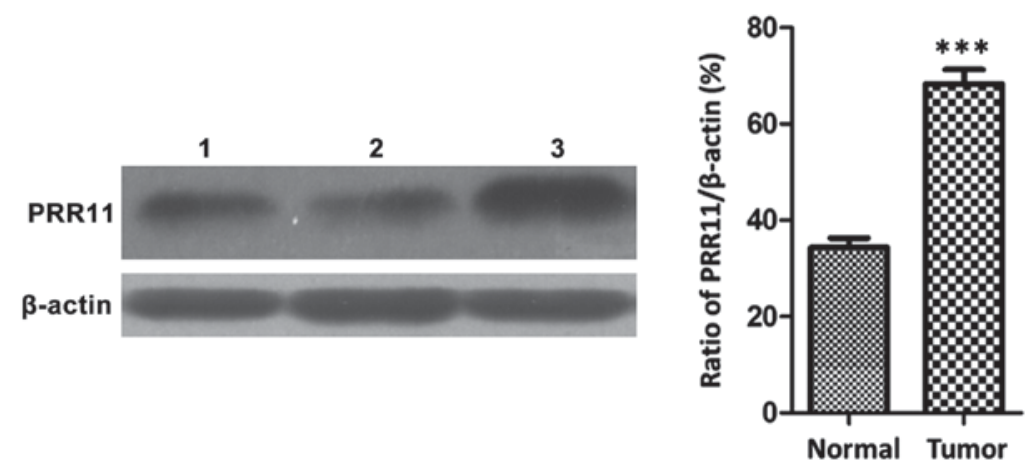

Figure 2. Western blot analysis for expression of PRR11 protein in pancreatic cancer tissues. Normal pancreatic tissue proteins on bands 1 and 2; pancreatic cancer tissue proteins on band $3 .{ }^{* * *} \mathrm{P}<0.001$.
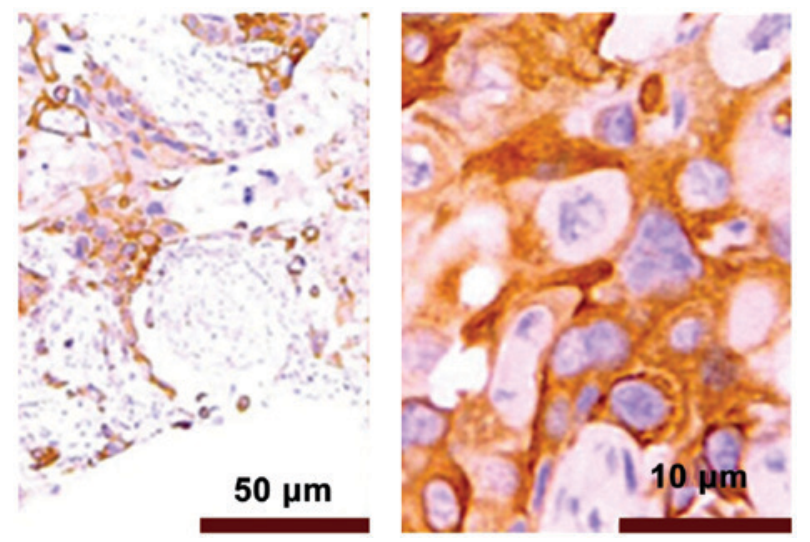

Figure 3. Immunohistochemistry for expression of PRR11 protein in normal pancreatic tissues (magnifications, x100 and x400).

cancer tissues was significantly higher than that in normal pancreatic tissues $(\mathrm{P}<0.01)$.

Expression level of PRR11 protein in pancreatic cancer tissues. The expression of PRR11 protein was measured in 38 cases of pancreatic cancer tissues and in 10 normal pancreatic tissues. Within the 38 cases of pancreatic cancer tissues, there were 30 positive cases by immunohistochemistry. In the 10 cases of normal tissues, the negative rate was $100 \%$, giving a $\mathrm{P}<0.05$ for a statistical significant difference. The results are shown in Table II.

Western blot results showed that the protein expression of PRR11 in pancreatic cancer tissues was significantly higher than that in non-cancerous tissues $(\mathrm{P}<0.001)$, as shown in Fig. 2.

The immunohistochemical results showed that PRR11 positive staining was pale yellow or brown yellow, as shown

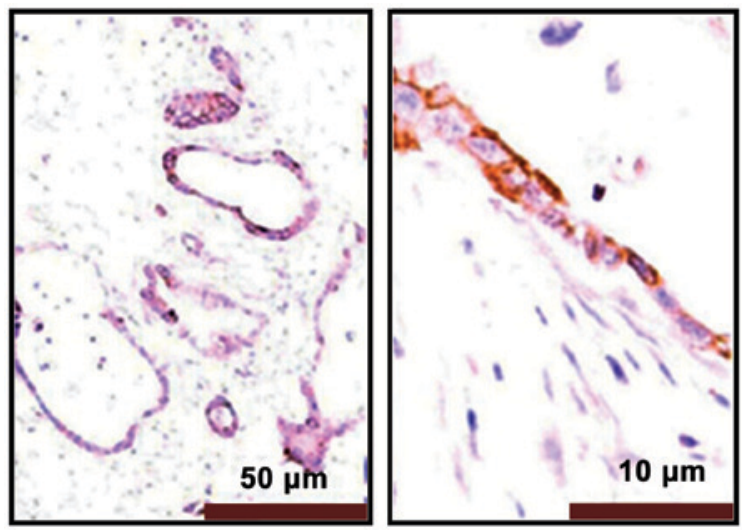

Figure 4. Comparison between the expression of PRR11 protein in normal pancreatic tissue and that of pancreatic cancer tissues detected by Immunohistochemistry (magnifications, x100 and x400).

in Fig. 3. Non-tumor tissues were non-stained or took on weak staining, as shown in Fig. 4.

Relationship between the expression of PRR11 protein and cell migration by the wound healing assay. After pPRR11-sh and pControl-sh cell strains were inoculated onto wells of a 6-well plate, photos were taken at 0,24 and $48 \mathrm{~h}$ to record the changes in cell migration rate. The results showed that the migration efficiency of the cancer cells with a PRR11 knockdown was obviously lower than that of the cancer cells expressing an empty control vector, indicating that inhibition of the expression of PRR11 may significantly reduce the cell migration. The results can be seen in Fig. 5.

Relationship between the expression of PRRII and clinical parameters. The differences in age and gender between patients with pancreatic cancer had no effects on the expression 
Table III. Relationship between the expression of PRR11 in pancreatic cancer and clinical parameters.

\begin{tabular}{|c|c|c|c|c|c|c|}
\hline Parameters & Cases & Positive & Negative & Positive rate (\%) & $\chi^{2}$ & P-value \\
\hline \multicolumn{7}{|l|}{ Age (years) } \\
\hline$\geq 60$ & 25 & 20 & 5 & 80.0 & & \\
\hline$<60$ & 13 & 10 & 3 & 76.9 & 1.013 & $\mathrm{P}=0.315$ \\
\hline \multicolumn{7}{|l|}{ Gender } \\
\hline Male & 20 & 16 & 4 & 80.0 & & \\
\hline Female & 18 & 14 & 4 & 77.8 & 0.058 & $\mathrm{P}=0.809$ \\
\hline \multicolumn{7}{|c|}{ Differentiation degree } \\
\hline High & 8 & 4 & 4 & 50.0 & & \\
\hline Middle & 18 & 14 & 4 & 77.78 & & \\
\hline Low & 12 & 12 & 0 & 100.0 & 8.881 & $\mathrm{P}=0.0118$ \\
\hline \multicolumn{7}{|l|}{ TNM stages } \\
\hline Stage 1 & 10 & 5 & 5 & 50.0 & & \\
\hline Stage 2 & 23 & 20 & 3 & 87.0 & & \\
\hline Stage 3 & 5 & 5 & 0 & 100.0 & 10.441 & $\mathrm{P}=0.0054$ \\
\hline \multicolumn{7}{|c|}{ Presence of lymphatic metastasis } \\
\hline Yes & 10 & 10 & 0 & 100.0 & & \\
\hline No & 28 & 20 & 8 & 71.4 & 9.305 & $\mathrm{P}=0.032$ \\
\hline
\end{tabular}
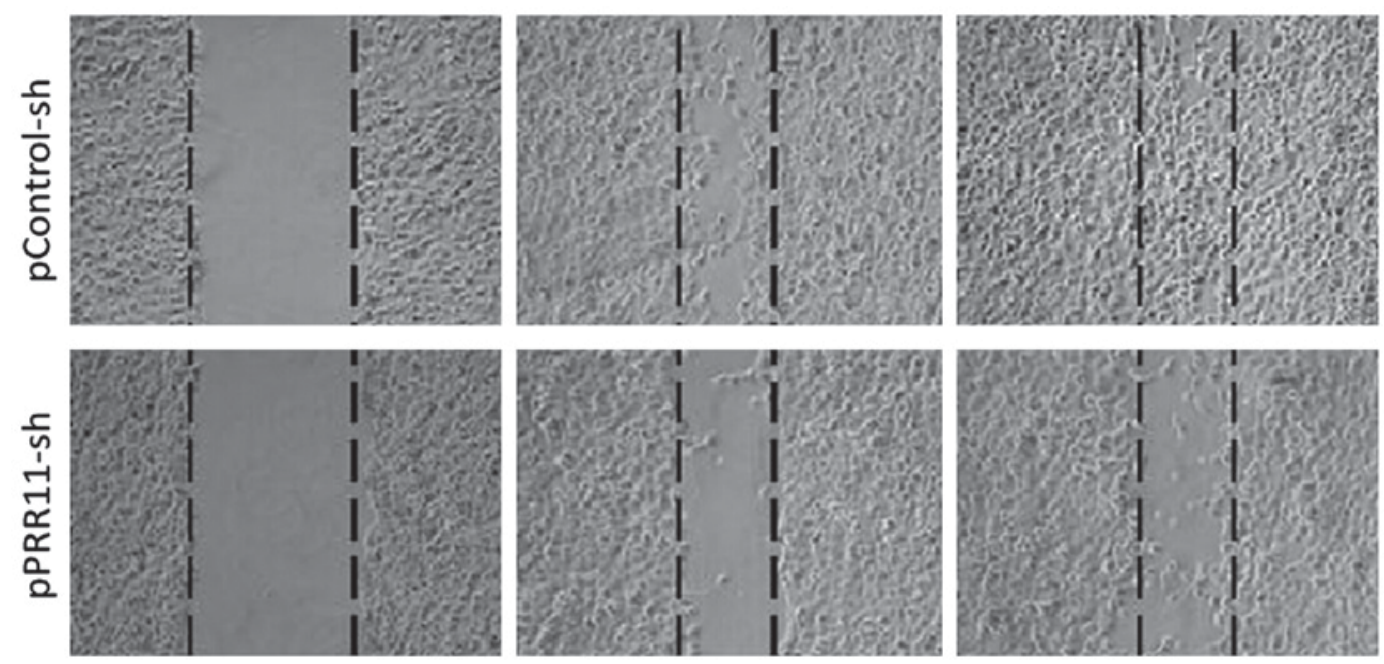

Figure 5. Wound healing assay using pancreatic cancer cells transfected with either pControl-sh or pPRR11-sh. The inhibition of the expression of PRR11 caused the reduction of the migration force of pancreatic cancer cells.

of PRR11 protein $(\mathrm{P}>0.05)$, while the differentiation degree, clinical TNM stages and lymphatic metastasis of pancreatic cancer cases were related to the expression of PRR11 protein $(\mathrm{P}<0.05)$. The results are shown in Table III.

Relationship between expression of PRR11 and prognosis. Follow-up was conducted for all the patients with pancreatic cancer. Their survival time was calculated from the completion of the operation. The clinical data of the selected cases were comprehensive and the cut-off time was March 2016. The analysis of survival data showed that there were 3 cases of failure to follow-up, 22 cases of death and 5 cases of survival. Among the death cases, 3 had negative expression and 19 had positive expression of PRR11 protein.
Among the survival cases, 4 cases had negative expression of PRR11 protein and 1 case had positive expression of PRR11 protein. A one-way analysis of variance was applied and a survival curve was drawn (Fig. 6). The survival time of negative expressors of PRR11 protein was clearly longer than that of positive expressors. The difference had statistical significance $(\mathrm{P}=0.0023)$ and the results are shown in Table IV and Fig. 6.

\section{Discussion}

Carrying a high mortality rate, pancreatic cancer is difficult to cure $(8,9)$. Moreover, its clinical manifestations are roughly similar to, and even the same as, those of other diseases of 
Table IV. Expression of PRR11 protein and its relation to the survival time of patients.

\begin{tabular}{lrcc}
\hline Groups & Cases & $\begin{array}{c}\text { Average survival } \\
\text { time (months) }\end{array}$ & P-value \\
\hline Negative PRR11 & 7 & 17.06 & \\
Positive PRR11 & 20 & 7.91 & $\mathrm{P}=0.0023$ \\
\hline
\end{tabular}

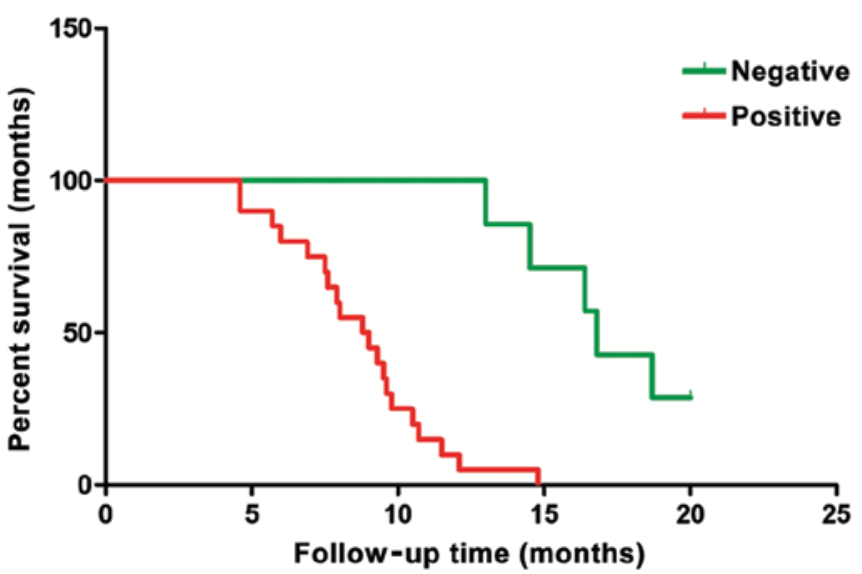

Figure 6. Survival curve of patients with pancreatic cancer.

the pancreas, which causes confusion amongst clinicians who cannot diagnose it accurately. At the same time, since the tumor deteriorates easily and the metastasis rate is high, the prognosis of the operation is not good. Researchers have hoped to obtain progress in the treatment methods by studying a large amount of cases and treatment protocols in the clinic. However, with the rapid development of molecular biology in recent years, that studies on pathological mechanisms at the molecular level are gaining more knowledge continuously. Some important genes and proteins influencing the occurrence and development of pancreatic cancer have been found (10-15). At present, PRR11 is a favorite marker in cancer studies as it has been proven to affect transcription of key players, which inhibit cancers and affect progress of various tumors. Studies have shown the PRR11 protein is mainly distributed in the cytoplasm and its gene is located in chromosome 17q22 (5). This gene has the highest expression level in ovaries and the thyroid gland and low expression levels in brain, heart and other organs. Its expression level in prostate, cervix uteri and lung tissues is slightly higher than that of other well-differentiated tissues. Based on this, many researchers have speculated that this gene may take part in the process of cell proliferation. Moreover, relevant studies on cancer tissues also have shown that PRR11 may cause a continuous deterioration of cancer, as its expression in malignant tumors is significantly higher than that in normal tissues $(16,17)$.

This study aimed at studying the expression of PRR11 protein in pancreatic cancer. Through analysis of our results, it was primarily observed that the prognosis of patients was related to the presence of absence of expression by immunohistochemistry. The expression of PRR11 protein in the
38 cases of human pancreatic cancer was higher than that in tissues from normal pancreas. The inhibition of the expression of PRR11 protein in pancreatic cancer cells reduced the cell migration capability of the cells, suggesting that the expression of PRR11 is related with the invasiveness of the pancreatic cancer.

The analysis of the follow-up data for the pancreatic cancer patients allowed us to conclude that the 5-year survival time for those with a PRR11 protein negative immunohistochemistry was higher than for those with high PRR11 expression. Moreover, it was found that the level of expression of the PRR11 protein in pancreatic cancer was positively correlated with invasion, disease stage and tissue differentiation of the tumor. This result was consistent with that reported in the literature. In our study, the average survival time of patients with positive expression of PRR11 protein was 8.52 months, while the average survival time of patients with negative expression was 16.23 months, suggesting a link between the level of PRR11 expression and the prognosis.

Collectively, this study showed that the levels of mRNA and protein from the PRR11 gene in the tissues of patients with pancreatic cancer were significantly higher than those in the tissues of normal patients. At least in theory, inhibiting the expression of the PRR11 gene could significantly inhibit the growth, proliferation, migration and tumorigenic ability of pancreatic cancer cells. Further studies should focus on the mechanism of action of PRR11 in the development of pancreatic cancer and other tumors, as well as on finding levels for diagnosis and treatment follow-up using PRR11 as a molecular target.

\section{References}

1. Luo J, Adami HO, Reilly M, Ekbom A, Nordenvall C and Ye W: Interpreting trends of pancreatic cancer incidence and mortality: a nation-wide study in Sweden (1960-2003). Cancer Causes Control 19: 89-96, 2008.

2. Beger HG, Rau B, Gansauge F, Leder G, Schwarz M and Poch B: Pancreatic cancer - low survival rates. Dtsch Arztebl Int 105: 255-262, 2008

3. Chen Y, Cha Z, Fang W, Qian B, Yu W, Li W, Yu G and Gao Y: The prognostic potential and oncogenic effects of PRR11 expression in hilar cholangiocarcinoma. Oncotarget 6: 20419-20433, 2015.

4. Ji Y, Xie M, Lan H, Zhang Y, Long Y, Weng H, Li D, Cai W, Zhu H, Niu Y, et al: PRR11 is a novel gene implicated in cell cycle progression and lung cancer. Int J Biochem Cell Biol 45: 645-656, 2013.

5. Song Z, Liu W, Xiao Y, Zhang M, Luo Y, Yuan W, Xu Y, Yu G and $\mathrm{Hu}$ Y: PRR11 is a prognostic marker and potential oncogene in patients with gastric cancer. PLoS One 10: e0128943, 2015.

6. Wang Y, Zhang Y, Zhang C, Weng H, Li Y, Cai W, Xie M, Long Y, Ai Q, Liu Z, et al: The gene pair PRR11 and SKA2 shares a NF-Y-regulated bidirectional promoter and contributes to lung cancer development. Biochim Biophys Acta 1849: 1133-1144, 2015.

7. Zhang C, Zhang Y, Li Y, Zhu H, Wang Y, Cai W, Zhu J, Ozaki T and $\mathrm{Bu}$ Y: PRR11 regulates late-S to $\mathrm{G} 2 / \mathrm{M}$ phase progression and induces premature chromatin condensation (PCC). Biochem Biophys Res Commun 458: 501-508, 2015.

8. Saif MW: Research in pancreatic cancer: an update after ASCO 2012. JOP 13: 330-331, 2012

9. Maisonneuve P and Lowenfels AB: Epidemiology of pancreatic cancer: an update. Dig Dis 28: 645-656, 2010.

10. Grønborg M, Kristiansen TZ, Iwahori A, Chang R, Reddy R, Sato N, Molina H, Jensen ON, Hruban RH, Goggins MG, et al: Biomarker discovery from pancreatic cancer secretome using a differential proteomic approach. Mol Cell Proteomics 5: 157-171, 2006. 
11. Stevens L,Pathak S, Nunes QM,Pandanaboyana S, Macutkiewicz C, Smart N and Smith AM: Prognostic significance of pre-operative C-reactive protein and the neutrophil-lymphocyte ratio in resectable pancreatic cancer: a systematic review. HPB Oxf 17: 285-291, 2015.

12. Kou T, Kanai M, Yamamoto M, Xue P, Mori Y, Kudo Y, Kurita A, Uza N, Kodama Y, Asada M, et al: Prognostic model for survival based on readily available pretreatment factors in patients with advanced pancreatic cancer receiving palliative chemotherapy. Int J Clin Oncol 21: 118-125, 2016.

13. Earl J, Garcia-Nieto S, Martinez-Avila JC, Montans J, Sanjuanbenito A, Rodríguez-Garrote M, Lisa E, Mendía E, Lobo E, Malats N, et al: Circulating tumor cells (Ctc) and kras mutant circulating free DNA (cfDNA) detection in peripheral blood as biomarkers in patients diagnosed with exocrine pancreatic cancer. BMC Cancer 15: 797, 2015.

14. Kishikawa T, Otsuka M, Ohno M, Yoshikawa T, Takata A and Koike K: Circulating RNAs as new biomarkers for detecting pancreatic cancer. World J Gastroenterol 21: 8527-8540, 2015.
15. Liu L, Xiang J, Chen R, Fu D, Hong D, Hao J, Li Y, Li J, Li S, Mou Y, et al; Chinese Study Group for Pancreatic Cancer (CSPAC): The clinical utility of CA125/MUC16 in pancreatic cancer: a consensus of diagnostic, prognostic and predictive updates by the Chinese Study Group for Pancreatic Cancer (CSPAC). Int J Oncol 48: 900-907, 2016.

16. Zhou F, Liu H, Zhang X, Shen Y, Zheng D, Zhang A, Lai Y and Li H: Proline-rich protein 11 regulates epithelial-to-mesenchymal transition to promote breast cancer cell invasion. Int J Clin Exp Pathol 7: 8692-8699, 2014.

17. Ho HY, Rohatgi R, Ma L and Kirschner MW: CR16 forms a complex with N-WASP in brain and is a novel member of a conserved proline-rich actin-binding protein family. Proc Natl Acad Sci USA 98: 11306-11311, 2001. 